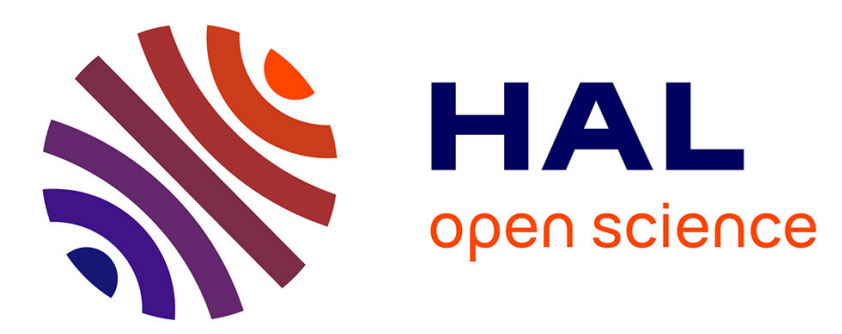

\title{
Counter approach for the estimation of optimal sequences in Partially Observable Untimed Petri Nets
}

Philippe Declerck

\section{To cite this version:}

Philippe Declerck. Counter approach for the estimation of optimal sequences in Partially Observable Untimed Petri Nets. Discrete Event Dynamic Systems, 2021, 31, pp.489-512. 10.1007/s10626-02100341-5 . hal-03185188

\section{HAL Id: hal-03185188 \\ https://univ-angers.hal.science/hal-03185188}

Submitted on 30 Mar 2021

HAL is a multi-disciplinary open access archive for the deposit and dissemination of scientific research documents, whether they are published or not. The documents may come from teaching and research institutions in France or abroad, or from public or private research centers.
L'archive ouverte pluridisciplinaire HAL, est destinée au dépôt et à la diffusion de documents scientifiques de niveau recherche, publiés ou non, émanant des établissements d'enseignement et de recherche français ou étrangers, des laboratoires publics ou privés. 


\title{
Counter approach for the estimation of optimal sequences in Partially Observable Untimed Petri Nets
}

\section{P. Declerck}

Received: date / Accepted: date

\begin{abstract}
In this paper, we consider the on-line estimation of optimal current subsequences in Partially Observable Untimed Petri Nets. Applying the counter approach classically used in max-plus algebra for Timed Petri nets, the idea is to exploit the assumption of a non immediate consumption of the tokens for each place which introduces an order of precedence between events. The approach can estimate a global price depending on the costs and gains provided by the tasks. The estimation of optimal sequences is based on the determination of a time horizon necessary to describe the sequences. The estimation is relevant to a step defined by two successive occurrences of observable transition firings. We show that the approach can consider any optimization problem if the dates of the observations are known or, if a guaranteed horizon can be computed which is always possible when the unobservable subnet satisfies a weak assumption close to the structural boundedness (relaxed structurally boundedness). As the technique avoids the generation of sets, the approach does not depend on their cardinalities and is numerically efficient.
\end{abstract}

Keywords Untimed Petri nets · Timed Petri nets · counter · partially observable $\cdot$ sequence estimation · sliding time horizon · relaxed structural boundedness

\section{Introduction}

The problem of estimating the state of a dynamic system is a fundamental issue in system theory. Observing a process and estimating its state consists in determining the value of a system variable from a certain set of measurements

P. Declerck

LISA/LARIS, University of Angers, 62 avenue Notre-Dame du Lac, 49000 Angers, France Tel.: +33241226560

Fax: +33241 226561

E-mail: philippe.declerck@univ-angers.fr 
given by a set of sensors. Contrary to [9] which considers Timed Labeled Petri nets, we focus on a untimed Petri net where by definition the time durations of the model are unknown; indeed, in some cases, the knowledge on the time durations can be partial or too complex to permit a resolution. The aim of this paper is to estimate possible firing sequences of unobservable transitions which are coherent with the observed label sequence produced by the observable transitions and present an optimal value for a criterion that is a linear weighting of the transition firing numbers. Generalizing the nonnegative cost studied in [16], this criterion can be a global price which is expressed with a function depending of the costs and the gains of the process.

Let us present the general context about untimed Petri nets and, particularly two main difficulties which must be tackled. Classically, the estimation approaches face an explosion of the numbers of the possible trajectories and markings which remains a challenge. The article [20] shows that the number of consistent markings in a Petri net with nondeterministic transitions (unobservable transitions and/or transitions that share the same label) is at most polynomial in the length of the observation sequence and so, increases with the new observations in the worst case which potentially leads to the stopping of the estimation at a given observation. Moreover, the number of firing sequences can be exponential in the length of the observation sequence.

Another limitation is the assumption of acyclic induced-subnet which is considered in many approaches and papers. Indeed, the estimation problem is often based on the generation of count vector giving a simplified picture of the evolution of the Petri net. As the firing conditions of the unobservable transitions are often neglected, the case of a count vector which does not represent a sequence is possible and the assumption of acyclic induced-subnet allows to remove this possibility: in that case, each count vector corresponds to at least a sequence and expresses a realistic behavior. However, this assumption limits the scope of the relevant results as a lot of processes are based on the repetition of tasks, which are modelled by circuits. Therefore, an objective is to generalize this hypothesis and to consider more general models containing circuits which can be self-loops.

In fact, the difficulty comes from the model of the Petri net which is composed of the fundamental equation of marking and an inequality expressing the firing condition of each transition. Contrary to the state equations used in automatic control or automata exploited in computer science, these two relations and their combination cannot easily be manipulated by standard tools and provide sequences. To overcome this drawback, a slight modification of the model is proposed in this paper: the idea is to introduce time in the model and precisely to associate with the untimed Petri net a new Timed Petri net where each place presents an elementary unitary temporization (Assumption $\mathcal{A S}-5$ ). As this time can be arbitrarily taken small, it implies that the consumption of the tokens for each place is not instantaneous. This weak modification of the Petri net creates a precedence between the events respectively associated with the input transitions and the output transitions and leads to the building of a polyhedron which completely describes the set of time sequences relevant 
to the checked count vector. Therefore, the estimation in the untimed case is based on a time model having a close behavior which allows to directly consider sequences. A consequence is that this variant of model and the relevant technique can consider any Petri net possibly with circuits and self-loops.

To describe sequences, we choose to use the "counter" form which represents the number of events at each moment and can describe complex structures with weights on the arcs [3] [17] [18]. Note that the "dater" form describes each event by a numbered date and can express complex synchronizations [10]; undoubtedly these two techniques have brought powerful approaches in the field of discrete event systems when the consideration of time is important [15].

Let us briefly give some other related approaches. The paper [2] gave a method for building the modified state class graph of a bounded time Petri net. The article [6] focused on diagnosability analysis of intermittent Faults. For the transitions sharing the same label and unobservable transitions, the study [20] determined bounds on the number of estimated markings and made the connections with the length of the observation sequence. As the estimation problem faces an explosion in the number of states, the approaches in [14] were based on a reduced observer which computes only a subset of the possible current markings for a partially observed Petri net. Based on [3], the study [18] presented an algebraic time model describing Timed Petri nets in standard algebra. Exploiting this study, the paper [9] adapted this model to the estimation problem and gave an algebraic form describing the complete solution set for a sequence of observed events without any assumption of acyclicity. This problem was also considered in [10] where the state space of the time event graphs with complex synchronizations such as P-time event graphs and time stream event graphs were expressed under a fixed-point form developed in the min-max-plus algebra. Standard algebra is considered in the estimation problem for P-time Petri nets [4]. Analogous to the well-known parity space used in fault detection for continuous systems, the generation of analytical redundancy relations for Timed Event Graphs in [11] avoided the state explosion since the technique based on an analogy of the Cayley Theorem in max-plus algebra would eliminate the state vector. The paper [1] focused on the same aim by applying the Fourier-Motzkin algorithm in partially observable Petri nets in the field of fault detection. While the objective of the paper [12] is a schedulability analysis of sequences relevant to candidate count vectors, this proposed paper follows another strategy as its aim is to directly provide sequences without searching candidate count vectors and to estimate a current majorant of the necessary horizon on-line.

In this paper, we assume the following assumptions for the untimed Petri net under investigation:

- Assumption $\mathcal{A S}-1$ : the incidence matrices and the initial marking (denoted $M^{\text {init }}$ below) are known.

- Assumption $\mathcal{A S}-2$ : the Petri net is live.

- Assumption $\mathcal{A S}-3$ : the occurrences of observable events are assumed to be non-simultaneous. 
- Assumption $\mathcal{A S}-4$ : the observations are distinguishable, that is, the same label cannot be associated with more than one observable transition.

The following assumptions are considered for the associated P-timed Petri net:

- Assumption $\mathcal{A S}-5$ : each place presents an elementary unitary temporization;

- Assumption $\mathcal{A S}-6$ : the dates of the successive observations are known. Assumption $\mathcal{A S}-6$ is considered in Section 3.2 and is removed in Section 4.

The assumption of boundedness of the marking and the hypothesis of acyclicity are not considered in this article contrary to many papers in this topic. We assume the presence of observations during the application of the estimation procedure as the estimation procedure exploits observations.

The paper is organized as follows. After the description of the preliminary notions in Section 2, Section 3 considers the description of P-timed Petri nets satisfying Assumption $\mathcal{A S}-5$. As article [9] indicated that time could be considered in an estimation problem on an arbitrary horizon with Assumption $\mathcal{A S}-6$, the objective is to adapt this approach to the untimed case for a current iteration $\langle k\rangle$ and to develop it on a relevant sliding horizon. The polyhedron which describes the estimation problem under an algebraic point of view and different criteria are presented. Then, Section 4 considers that the case where Assumption $\mathcal{A S}-6$ cannot be taken. This section focuses on the computation and analysis of a guaranteed sliding horizon, that is, an horizon which is sufficiently large such that any possible sequence is modelled in the estimation problem. The proposed approach is illustrated by a pedagogical example containing an unobservable circuit in Section 5. Finally, a comparison with other approaches is made in Section 6.

\section{Preliminary}

\subsection{Notations}

The notation $|Z|$ is the cardinality of set $Z$ and the notation $A^{T}$ corresponds to the transpose of matrix $A$. The 1 -norm of vector $u$ is equal to the sum of the absolute values of the vector elements and is denoted $\|u\|_{1}$. The notation $\lfloor x\rfloor$ represents the greatest integer less than or equal to $x$. A Place/Transition $(\mathrm{P} / \mathrm{TR})$ net is the structure $N=\left(P, T R, W^{+}, W^{-}\right)$, where $P$ is a set of $|P|$ places and $T R$ is a set of $|T R|$ transitions. The matrices $W^{+}$and $W^{-}$are respectively the $|P| \times|T R|$ post and pre-incidence matrices over $\mathbb{N}$, where each row $l \in\{1, \ldots,|P|\}$ specifies the weight of the incoming and outgoing arcs of the place $p_{l} \in P$. The incidence matrix is $W=W^{+}-W^{-}$. The preset and postset of the node $v \in P \bigcup T R$ are denoted by $\bullet v$ and $v^{\bullet}$, respectively. The notation $\Omega^{*}$ represents the set of firing sequences, denoted $\sigma$, consisting of transitions of the set $\Omega \subset T R$. The vector $\bar{\sigma}$ of dimension $|T R|$ expresses the firing vector or count vector of the sequence $\sigma \in T R^{*}$, where the $i$-th 
component $\overline{\sigma_{i}}$ is the firing number of the transition $t r_{i} \in T R$ which is fired $\overline{\sigma_{i}}$ times in the sequence $\sigma$.

The marking of the set of places $P$ is a vector $M \in \mathbb{N}^{|P|}$ that assigns to each place $p_{i} \in P$ a non-negative integer number of tokens $M_{i}$, represented by black dots. The $i$-th component $M_{i}$ is also written $M\left(p_{i}\right)$. The marking $M$ reached from the initial marking $M^{\text {init }}$ (which replaces the usual notation $M_{0}$ ) by firing the sequence $\sigma$ can be calculated by the fundamental relation: $M=M^{i n i t}+W \cdot \bar{\sigma}$. The transition $t r$ is enabled at $M$ if $M \geq W^{-}(., t r)$ and may be fired yielding the marking $M^{\prime}=M+W(., t r)$. We write $M[\sigma \succ$ to denote that the sequence of transitions $\sigma$ is enabled at $M$, and we write $M\left[\sigma \succ M^{\prime}\right.$ to denote that the firing of $\sigma$ yields $M^{\prime}$.

\subsection{Definitions}

Definition 1 A Petri net is said to be bounded if the number of tokens in each place does not exceed a finite natural number for any marking reachable from the initial marking.

Definition 2 A Petri net is said to be Structurally Bounded (SB) if and only if there exists a vector $z$ with positive integer entries such that $z . W \leq 0$. Formally,

$$
\exists z>0 \text { such that } z \cdot W \leq 0
$$

Definition 3 A Petri net is said to be deadlock structurally bounded (DSB) if and only if there exists a vector $z$ with positive integer entries such that $z . W<0$. Formally,

$$
\exists z>0 \text { such that } z . W<0
$$

Note that a DSB net is not live as it leads to a deadlock state. Let us take a vector $z>0$ such that $z . W<0$. If we have some firings expressed by the count vector $\bar{\sigma}$, we have $M^{\text {init }}+W \cdot \bar{\sigma}=M$. So, $z \cdot M^{i n i t}+z \cdot W \cdot \bar{\sigma}=z \cdot M$ $\Rightarrow z \cdot M^{i n i t}>z \cdot M$. Another evolution described by $\overline{\sigma^{\prime}}$ gives $M+W \cdot \overline{\sigma^{\prime}}=M^{\prime}$ which also gives $z \cdot M>z \cdot M^{\prime}$. Finally, $z \cdot M^{\text {init }}>z \cdot M>z \cdot M^{\prime}>\ldots$ and, as the inequalities are strict, the nonnegative product converges to zero which is a deadlock state.

\subsection{First notations for estimation}

A labeling function $L: T R \rightarrow A L \cup\{\varepsilon\}$ assigns to each transition $\operatorname{tr} \in T R$ either a symbol from a given alphabet $A L$ or the empty string $\varepsilon$. In a partially observed Petri net, we assume that the set of transitions $T R$ can be partitioned as $T R=T R_{\text {obs }} \cup T R_{u n}$, where the set $T R_{o b s}$ (respectively, $T R_{u n}$ ) is the set of observable transitions associated with a label of $A L$ (the empty string $\varepsilon$ ). 
In this paper, we assume that the same label of $A L$ cannot be associated with more than one transition of $T R_{o b s}$.

The $T R_{u n}$-induced subnet of the Petri net $N$ is defined as the new net $N_{u n}=\left(P, T R_{u n}, W_{u n}^{+}, W_{u n}^{-}\right)$, where $W_{u n}^{+}$and $W_{u n}^{-}$(respectively, $W_{o b s}^{+}$and $\left.W_{o b s}^{-}\right)$are the restrictions of $W^{+}$and $W^{-}$to $P \times T R_{u n}$ (respectively, $P \times$ $\left.T R_{o b s}\right)$. Therefore, $W_{u n}=W_{u n}^{+}-W_{u n}^{-}\left(\right.$respectively, $\left.W_{o b s}=W_{o b s}^{+}-W_{o b s}^{-}\right)$. A reorganization of the columns with regards to $T R_{u n}$ and $T R_{o b s}$ yields $W=$ $\left(W_{u n} W_{o b s}\right)$. Notation $x_{i}$ expresses an unobservable transition, belonging to $T R_{u n}$ while an observable transition belonging to $T R_{o b s}$ is denoted $y_{i}$.

The notation of the count vectors is taken for $\bar{x}$ of dimension $\left|T R_{u n}\right|$ and $\bar{y}$ of dimension $\left|T R_{o b s}\right|$. The reorganization of the components of $\bar{\sigma}$ yields $\bar{\sigma}=\left(\bar{x}^{T} \bar{y}^{T}\right)^{T}$.

In this paper, the time is discrete and the occurrence of each event is synchronized with an external clock. The origin of time zero is absolute, that is, common to all dates considered in this paper. Starting from the marking $M^{<1>}$ which is the initial marking $M^{\text {init }}$ at time zero, the estimation of the current unobservable sequence is based on the treatment of the data produced by the observed transitions successively in an on-line procedure at the dates $t^{<1>}, t^{<2>}, t^{<3>}, \ldots$ where: $t^{<k>}$ is the $k t h$ firing date of an observable transition which is defined with respect to the origin of time $t^{<0>}=0$; moreover, $\langle k>$ is the $k t h$ step relevant to a time interval $\left[t^{<k-1>}, t^{<k>}\right]$ for $k \geq 1$. By construction, $t^{<k-1>} \neq t^{<k>}$ for $k \geq 1$ and $t^{<1>}$ is relevant to the first observation. The notation $\bar{y}^{<k>}$ represents the count vector of observable transitions firing at time $t^{<k>}$ exactly and Assumption $\mathcal{A S}-3$ implies $\left\|\bar{y}^{<k>}\right\|_{1}=1$. Notation $\bar{x}^{<k>}$ represents the count vector for the unobservable transitions $T R_{u n}$ for step $<k>$. From $M^{<k>}$, the transition firings relevant to $\bar{x}^{<k>}$ and $\bar{y}^{<k>}$ allow the establishment of marking $M^{<k+1>}$ : formally, $M^{<k>}\left[\sigma^{<k>} \succ M^{<k+1>}\right.$ such that $\bar{\sigma}^{<k>}=\left(\left(\bar{x}^{<k>}\right)^{T}\left(\bar{y}^{<k>}\right)^{T}\right)^{T}$. As $M^{<1>}$ is the initial marking relevant to time $t^{<0>}=0$, we assume that $\bar{x}^{<k>}=0$ and $\bar{y}^{<k>}=0$ for $k \leq 0$. So, the estimation must consider $M^{<1>}\left[x^{<1>} y^{<1>} \succ M^{<2>}\right.$ for step $<1>$ where the time horizon is $\left[t^{<0>}, t^{<1>}\right]$ and $t^{<1>}$ is relevant to the first observation $y^{<1>}$, then $M^{<2>}\left[x^{<2>} y^{<2>} \succ M^{<3>}\right.$ for step $<2>$ where the time horizon is $\left[t^{<1>}, t^{<2>}\right]$ and $t^{<2>}$ is relevant to the second observation $y^{<2>}$, and so on. Note that these notations are not cumulative as we can have $\bar{x}^{<3>}=0$ but $\bar{x}^{<1>} \neq 0$ and $\bar{x}^{<2>} \neq 0$ : the condition $\bar{x}^{<1>} \leq \bar{x}^{<2>} \leq \bar{x}^{<3>}$ does not hold.

\section{Estimation of sequences}

\subsection{Aim and principle of the approach}

The problem considered in the paper is as follows. Let us consider a untimed Petri net where the incidence matrix $W$ and the initial marking $M^{<1>}$ are known. We focus on the subsequences of the unobservable firing events of the transitions of $T R_{u n}$ for each step $\langle k\rangle$. Given a sequence of the observed 
firing events of the transitions of $T R_{o b s}$ generated by the activity of the Petri net, we want to find the relevant subsequences of the unobservable firing events of the transitions of $T R_{u n}$ for each step $<k>$ which are optimal with respect to a general criteria given in the sequel.

Let us present the principle of the approach. The system of relations describing a Petri net (that is, the fundamental equation of marking and the firing condition of the transitions) does not express a precedence between the events in a simple way. To compensate this drawback, the idea is to introduce a precedence between the events by slightly modifying the Petri net and considering a specific count vector. As a sequence is a succession of events, we take the assumption that the consumption of the tokens for each place is not instantaneous. In that aim, we associate a temporization equal to an elementary time unit with each place, which implies that the input flow does not present an immediate effect on the output flow (Assumption $\mathcal{A S}-5$ ). Let us consider the case of a unitary valuation on the arcs. If an input transition is fired, then a token is produced, but no output transition immediately consumes this produced token. Therefore, this time model distinguishes some firings of the input and output transitions (this point is illustrated by Example 2 below).

In that case, the model is more realistic as the possible infinite sequences in untimed Petri nets disappear for the timed Petri nets. In other words, the length of any sequence is finite. Precisely, the infinite number of events of these sequences is replaced by an arbitrary large number of events: this transformation is usually made in linear programming to treat infinite values. Note that an infinite sequence does not seem to represent a realistic evolution as it corresponds to an infinite number of train arrival at a station or an infinite production by a machine.

\subsection{Polyhedron}

As the modelling of time must be made, the previous notations must be completed with the following notations which are cumulative from the origin of time. Each transition $x_{i}$ is associated with the number of events that happen before or at time $\Theta$ and is denoted $\overline{x_{i}(\Theta)}$. Assuming that the events can only occur at $\Theta \geq 1$, we have $x(\Theta)=0$ for $\Theta \leq 0$.

Example 1. Let us show the possibilities of this notation. Note that for a given transition $i$, if there are two different instants $\Theta_{1}$ and $\Theta_{2}$ such that $\overline{x_{i}\left(\Theta_{1}\right)} \neq \overline{x_{i}\left(\Theta_{2}\right)}$, then we can conclude the occurrence of an event at least. Indeed, the arrival of two events at times 3 and 5 implies that the sequence of numbers of events starting at $\Theta=0$ and finishing at $\Theta=7$ is $0,0,0,1,1,2,2,2, \ldots$ that is $\overline{x_{i}(\Theta=3)}=1$ and $\overline{x_{i}(\Theta=5)}=2$ but also $\overline{x_{i}(\Theta=4)}=1$ and $\overline{x_{i}(\Theta=7)}=2$. The series of number of events $\overline{x_{i}(\Theta)}$ can express different situations: no event may take place at time $\Theta\left(\overline{x_{i}(\Theta=3)}=1\right.$ and $\overline{x_{i}(\Theta=4)}=1$ describes the absence of event for $\left.\Theta=4\right)$; a single event may happen at $\Theta \overline{\left(x_{i}(\Theta=4)\right.}=1$ and $\overline{x_{i}(\Theta=5)}=2$ represents one event at $\left.\Theta=5\right)$; 
several events may occur simultaneously at $\Theta$ (the sequence $0,1,5, \ldots$ expresses 4 events at $\Theta=3$ and so, we consider a multi-server semantic with a unique variable $\overline{x_{i}(\Theta)}$.

The above notions are usual in max-plus algebra but must be adapted to the problem. Let $\overline{y(\theta)}$ (respectively, $\overline{x(\theta)}$ ) be the count subvector of the state vector $x(\theta)$ at time $\theta$ such that the relevant transitions belong to the set of observable transitions $T R_{\text {obs }}$ (respectively, unobservable transitions $T R_{u n}$ ).

The on-line diagnosis procedure is based on a sliding horizon which is relevant to a current step $\langle k\rangle$ for $k \geq 1$ separating two successive dates of observations $t^{<k-1>}, t^{<k>}$ of the last successive observations $y^{<k-1>}, y^{<k>}$.

Step $<0>$ corresponds to the initialization with $\bar{x}^{<0>}=0$ and $\bar{y}^{<0>}=0$ while the state computation starts at step $<1>$. We take the weak Assumption $\mathcal{A S}-6$ that the two dates at step $\langle k\rangle$ are known and we can define a time horizon

$$
h^{<k>}=t^{<k>}-t^{<k-1>}
$$

relevant to step $\left\langle k>\right.$. For simplicity of the writing, $h^{<k>}$ and $t^{<k>}$ are denoted $h$ and $t$ respectively. We take:

$$
\begin{aligned}
& \bar{\gamma}=\left((\overline{y(t-h)})^{T}(\overline{y(t-h+1)})^{T} \ldots(\overline{y(t-1)})^{T}(\overline{y(t)})^{T}\right)^{T} \text { and } \\
& \bar{\varkappa}=\left((\overline{x(t-h)})^{T}(\overline{x(t-h+1)})^{T} \ldots\right. \\
&(\overline{x(t-1)})^{T} \underline{\left.(\overline{x(t)})^{T}\right)^{T} .}
\end{aligned}
$$

Contrary to $\overline{y(\theta)}$, the notation $\bar{y}^{<k>}$ introduced at the previous section is not cumulative on all past iterations, and so, $\overline{y(t)} \underset{k^{\prime}=1, \ldots, k}{=\sum^{<k^{\prime}>}}$. The introduction of the notation $\bar{y}^{<0>\rightarrow<k>} \underset{k^{\prime}=1, \ldots, k}{=\sum^{<k^{\prime}>}}$ allows to give short expressions of the known data for the iteration $<k>$ which are:

$$
\begin{aligned}
& \overline{y(t-h)}=\bar{y}^{<0>\rightarrow<k-1>} \\
& \overline{y(t)}=\bar{y}<0>\rightarrow<k>
\end{aligned}
$$

and $\bar{y}^{<k>}=\overline{y(t)}-\overline{y(t-h)}$. By construction, there is no firing of an observable transition in step $\langle k\rangle$ for $\theta \in\{t-h+1, t-h+2, \ldots, t-1\}$ and formally, $\overline{y(\theta)}=\overline{y(t-h)}=\bar{y}^{<0>\rightarrow<k-1>}$ for $t-h+1 \leq \theta \leq t-1$.

Similarly, the notation $\overline{x(\theta)}$ ) is cumulative on all past iterations contrary to $\bar{x}^{<k>}$ and particularly $\overline{x(t)}=\sum_{k^{\prime}=1, \ldots, k} \bar{x}^{<k^{\prime}>}$. The notation $\bar{x}^{<0>\rightarrow<k>}=$ $\sum_{k^{\prime}=1, \ldots, k} \bar{x}^{<k^{\prime}>}$ allows to write

$$
\begin{aligned}
& \overline{x(t-h)}=\bar{x}^{<0>\rightarrow<k-1>} \\
& \overline{x(t)}=\bar{x}^{<0>\rightarrow<k>}
\end{aligned}
$$

and $\bar{x}^{<k>}=\overline{x(t)}-\overline{x(t-h)}$.

The evolution can be described by the following inequalities expressing the relations between the firing event numbers of transitions. For each place $p_{l} \in P$ which is associated with a time duration $\Delta_{l}$, we can write that the output flow of tokens at time $\theta \in\{t-h+1, t-h+2, \ldots, t\}$ is lower than or equal to the 
addition of the initial marking of $p_{l}$, that is, $M_{l}^{\text {init }}$, and the input flow with a delay $\Delta_{l}$.

$$
W_{l, .}^{-} \overline{x(\theta)} \leq W_{l, .}^{+} \overline{x\left(\theta-\Delta_{l}\right)}+M_{l}^{\text {init }} .
$$

\section{Example 2}

The Timed Petri net in Fig. 1 allows to illustrate relation (3). The place $p_{1}$ with time duration $\Delta_{1}$ in Fig. 1 is described by inequality

$$
\overline{x_{3}(\theta)}+\overline{x_{4}(\theta)} \leq \overline{x_{1}\left(\theta-\Delta_{1}\right)}+\overline{x_{2}\left(\theta-\Delta_{1}\right)}+M_{1}^{\text {init }}
$$

and so, $W_{1, .}^{-}=\left(\begin{array}{llll}0 & 0 & 1 & 1\end{array}\right)$ and $W_{2, .}^{+}=\left(\begin{array}{llll}1 & 1 & 0 & 0\end{array}\right)$.

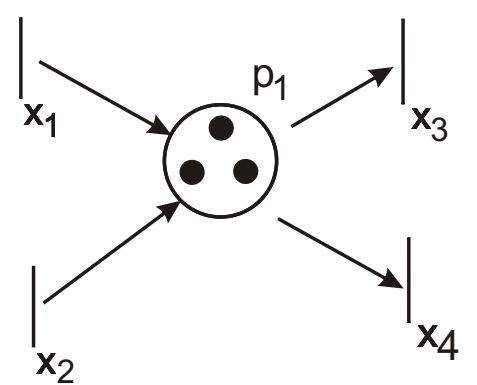

Fig. 1 A place of a Petri net (example 2)

We now consider Assumption $\mathcal{A S}-5$, that is, time duration $\Delta_{l}$ is unitary. The introduction of this assumption leads to a weak restriction of the behavior of the Petri net about the firings of the input and output transitions when there is a causal connection between the relevant events. Precisely, when the valuations of the Petri net are unitary, the above expression expresses that the sum of the firing numbers of the output transitions is lower than or equal to the sum of firing numbers of the input transitions for each place at a given time $\theta$ and the initial marking. The valuated case is more complex as it introduces ponderations in the sums. The following example shows that Assumption $\mathcal{A S}-5$ implies that some events cannot be simultaneous.

Example 2 continued.

A simple interpretation can be presented if a simplification of Example 2 is made. We consider the Timed Petri net of Fig. 1 presenting a unique place $p_{1}$ with $\Delta_{1}=1$ but transitions $x_{2}$ and $x_{4}$ are deleted $\left(|\bullet p|=\left|p^{\bullet}\right|=1\right)$ and the tokens of $p$ are removed $\left(M_{1}^{\text {init }}=0\right)$ : so, we can write $\overline{x_{3}(\theta+1)} \leq \overline{x_{1}(\theta)}$ with $\overline{x_{1}(\theta)}=\overline{x_{3}(\theta)}=0$ for $\theta \leq 0$. If $\overline{x_{1}(\theta=0)}$, then $\overline{x_{3}(\theta=1)}=0$. If the first firing of $x_{1}$ occurs at $\theta=1$, we have $\overline{x_{1}(\theta=1)}=1$ and $\overline{x_{3}(\theta=2)}=0$ or $\overline{x_{3}(\theta=2)}=1$. Therefore, the first firing of $x_{3}$ which can occur at $\theta=2$ cannot be simultaneous with the first firing of $x_{1}$. So, the $k$ th firing date of an output transition of a place occurs strictly after the $k t h$ firing date of the relevant 
input transition and the relevant events are not simultaneous. Now, a second firing of $x_{1}$ can occur at $\theta=2\left(\overline{x_{1}(\theta=2)}=2\right)$ and, if $\overline{x_{3}(\theta=2)}=1$, then the two relevant events are simultaneous. Therefore, in a Timed Event Graph with a null initial marking, the $k t h$ firing date of the input and output transitions cannot be simultaneous but this rule depends on the firing number $k$. If the initial marking is non-null, a shift in the numbering must be introduced in the interpretation.

After an adequate permutation of the columns of matrices, the consideration of the observable/unobservable transitions gives

$$
\begin{gathered}
W_{u n}^{-} \cdot \overline{x(\theta)}+W_{o b s}^{-} \cdot \overline{y(\theta)} \leq W_{u n}^{+} \cdot \overline{x\left(\left(\theta-\Delta_{l}\right)\right)}+ \\
W_{o b s}^{+} \cdot \overline{y\left(\left(\theta-\Delta_{l}\right)\right)}+M^{<1>}
\end{gathered}
$$

for the set of places $P$ with $\Delta_{l}=1$ and we obtain the system

$$
\mathbf{A}_{1} \cdot \bar{\varkappa} \leq \mathbf{C}_{1}-\mathbf{B}_{1} \cdot \bar{\gamma}
$$

where

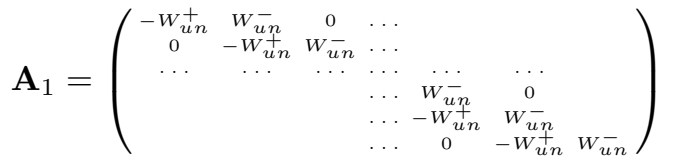

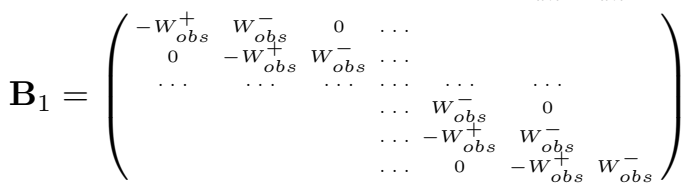

$$
\begin{aligned}
& \text { and } \mathbf{C}_{1}=\left(\begin{array}{c}
M^{<1>} \\
M^{<1>} \\
\ldots \\
M^{<1>} \\
M^{<1>} \\
M^{<1>}
\end{array}\right) \text {. }
\end{aligned}
$$

Note that vector $\mathbf{C}_{1}$ does not depend on the current marking which brings an important advantage.

The dimension of vector $\bar{\varkappa}$ is denoted by $n=(h+1) \cdot\left|T R_{u n}\right|$ while the dimension of vector $\bar{\gamma}$ is $(h+1) .\left|T R_{o b s}\right|$. The dimensions of matrices $\mathbf{A}_{1}, \mathbf{B}_{1}$, $\mathbf{C}_{1}$ and column vector $\mathbf{b}_{1}=\mathbf{C}_{1}-\mathbf{B}_{1} \cdot \bar{\gamma}$ are respectively $(h \cdot|P| \mathbf{x} n),(h .|P| \mathbf{x}$ $\left.(h+1) \cdot\left|T R_{o b s}\right|\right),(h .|P| \times 1)$ and $(h \cdot|P| \times 1)$.

The unobservable trajectory $\bar{\varkappa}$ is non-decreasing and we must add the condition

$$
\overline{x(\theta-1)} \leq \overline{x(\theta)}
$$


for $\theta \in\{t-h+1, t-h+2, \ldots, t\}$ or $\mathbf{A}_{2}=\left(\begin{array}{cccccc}I & -I & 0 & \ldots & & \\ 0 & I & -I & \ldots & & \\ \ldots & \ldots & \ldots & \ldots & \ldots & \\ & \ldots & -I & 0 \\ & & \ldots & I & -I \\ & & \ldots & 0 & I & -I\end{array}\right) \mathbf{b}_{2}=$ $\left(\begin{array}{c}0 \\ 0 \\ \cdots \\ 0 \\ 0 \\ 0\end{array}\right)$. The dimensions of matrices $\mathbf{A}_{2}$ and column vector $\mathbf{b}_{2}$ are respectively
$\left(h .\left|T R_{u n}\right| \times n\right)$ and $\left(h \cdot\left|T R_{u n}\right| \times 1\right)$. Moreover, we have

$$
\mathbf{A}_{3} \cdot \bar{\varkappa} \leq \mathbf{b}_{3}
$$

where $\mathbf{A}_{3}=-I_{n \times n}$ and $\mathbf{b}_{3}=0_{n \times 1}$ as the trajectories are non-negative.

Finally, a synthesis of the previous systems (5) (6) and (7) allows to obtain a simple polyhedron of the general form

$$
\mathbf{A} \cdot \bar{\varkappa} \leq \mathbf{b}^{<k>}
$$

with $\mathbf{A}=\left(\begin{array}{c}\mathbf{A}_{1} \\ \mathbf{A}_{2} \\ \mathbf{A}_{3}\end{array}\right)$ and $\mathbf{b}^{<k>}=\left(\begin{array}{c}\mathbf{b}_{1}=\mathbf{C}_{1}-\mathbf{B}_{1} \cdot \bar{\gamma} \\ \mathbf{b}_{2} \\ \mathbf{b}_{3}\end{array}\right)$

which allows to express the unobservable sequences of the Petri nets for the iteration $\langle k\rangle$.

\section{Example 3.}

Let us build systems (5) (6) and (7) for the Timed Petri net of Fig. 2.

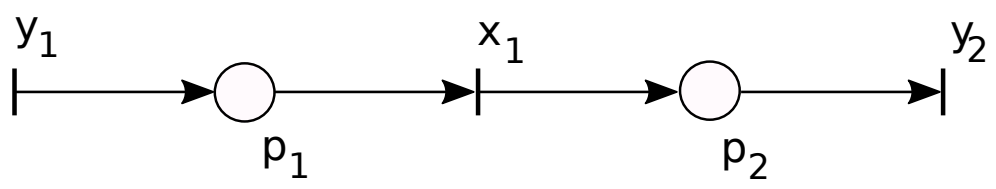

Fig. 2 Petri net with unitary time durations (example 3)

All the places of the P-timed Petri net of Fig. 2 present unitary time durations. The relevant relations are $\left\{\begin{array}{l}\overline{x_{1}(\theta)} \leq \overline{y_{1}(\theta-1)}+M_{1}^{<1>} \\ \overline{y_{2}(\theta)} \leq \overline{x_{1}(\theta-1)}+M_{2}^{<1>}\end{array}\right.$ with $\overline{x_{1}(\theta-1)} \leq$ $\overline{x_{1}(\theta)}$ and $\overline{x_{1}(\theta)} \geq 0$ for $\theta \in\left\{t^{<k>}-h, t-h+2, \ldots, t^{<k>}\right\}$. For an arbitrary horizon $h=3$, we have

$\bar{\gamma}=\left((\overline{y(t-3)})^{T}(\overline{y(t-2)})^{T}(\overline{y(t-1)})^{T}(\overline{y(t)})^{T}\right)^{T}$ and $\left.\bar{\varkappa}=\left(\overline{\left(x_{1}(t-3)\right.}\right)^{T}\left(\overline{x_{1}(t-2)}\right)^{T}\left(\overline{x_{1}(t-1)}\right)^{T}\left(\overline{x_{1}(t)}\right)^{T}\right)^{T}$. As $\theta \in\{t-2, t-1, \ldots, t\}$ 
with $t=t^{<k>}$, the relations (5) are $\left\{\begin{aligned} & \overline{x_{1}(t-2)} \leq \overline{y_{1}(t-3)}+M_{1}^{<1>} \\ & \overline{x_{1}(t-3)} \leq \overline{y_{2}(t-2)}+M_{2}^{<1>} \\ & \overline{x_{1}(t-1)} \leq \overline{y_{1}(t-2)}+M_{1}^{<1>} \\ & \overline{x_{1}(t-2)} \leq-\overline{y_{2}(t-1)}+M_{2}^{<1>} \\ & \overline{x_{1}(t)} \leq \overline{y_{1}(t-1)}+M_{1}^{<1>} \\ &-\overline{x_{1}(t-1)} \leq-\overline{y_{2}(t)}+M_{2}^{<1>}\end{aligned}\right.$ or

$\left(\begin{array}{cccc}0 & 1 & 0 & 0 \\ -1 & 0 & 0 & 0 \\ 0 & 0 & 1 & 0 \\ 0 & -1 & 0 & 0 \\ 0 & 0 & 0 & 1 \\ 0 & 0 & -1 & 0\end{array}\right) . \bar{\varkappa} \leq\left(\begin{array}{cccccccc}1 & 0 & 0 & 0 & 0 & 0 & 0 & 0 \\ 0 & 0 & 0 & -1 & 0 & 0 & 0 & 0 \\ 0 & 0 & 1 & 0 & 0 & 0 & 0 & 0 \\ 0 & 0 & 0 & 0 & 0 & -1 & 0 & 0 \\ 0 & 0 & 0 & 0 & 1 & 0 & 0 & 0 \\ 0 & 0 & 0 & 0 & 0 & 0 & 0 & -1\end{array}\right) . \bar{\gamma}+\left(\begin{array}{l}M_{1}^{<1>} \\ M_{2}^{<1>} \\ M_{1}^{<1>} \\ M_{2}^{<1>} \\ M_{1}^{<1>} \\ M_{2}^{<1>}\end{array}\right)$.

Inequality (6) becomes:

$\overline{x_{1}(t-3)} \leq \overline{x_{1}(t-2)} \leq \overline{x_{1}(t-1)} \leq \overline{x_{1}(t)}$ or

$\left\{\begin{array}{l}\overline{x_{1}(t-3)}-\overline{x_{1}(t-2)} \leq 0 \\ \overline{x_{1}(t-2)}-\overline{x_{1}(t-1)} \leq 0 \\ \overline{x_{1}(t-1)}-\overline{x_{1}(t)} \leq 0\end{array}\right.$ which is $\left(\begin{array}{rrrr}1 & -1 & 0 & 0 \\ 0 & 1 & -1 & 0 \\ 0 & 0 & 1 & -1\end{array}\right) \cdot \bar{\varkappa} \leq 0$

System (7) is expressed by:

$\overline{x_{1}(t-3)} \geq 0, \overline{x_{1}(t-2)} \geq 0, \overline{x_{1}(t-1)} \geq 0, \overline{x_{1}(t)} \geq 0$ or $\left(\begin{array}{cccc}-1 & 0 & 0 & 0 \\ 0 & -1 & 0 & 0 \\ 0 & 0 & -1 & 0 \\ 0 & 0 & 0 & -1\end{array}\right) . \bar{\varkappa} \leq$

0 .

\subsection{Criteria}

Let us present some criteria $c . \bar{\varkappa}$ which can be exploited in the optimization with system (8).

- If we want to estimate a sequence inside the step $\langle k\rangle$ presenting the minimum or maximum number of events for the Petri net, we can express the sum of the event numbers with $c_{1 \mathrm{x}\left|T R_{u n}\right|} \mid \bar{x}^{<k>}$ where $c_{1 \mathrm{x}\left|T R_{u n}\right|}$ is unitary. As $\bar{x}^{<k>}=\overline{x(t)}-\overline{x(t-h)}$, we can compute the difference between $(\overline{x(t)})$ and $(\overline{x(t-h)})$ by taking $c_{d i f f} \cdot \bar{\varkappa}$ (diff: difference) and $c_{d i f f}=$ $\left(-c_{1 \mathrm{x}\left|T R_{u n}\right|} 0 \ldots 0 c_{1 \mathrm{x}\left|T R_{u n}\right|}\right)$ where $c_{1 \mathrm{x}\left|T R_{u n}\right|}$ is unitary.

- We can consider that each task is expressed by the presence of a token in a place associated with a cost (produced by the amount of products or energy needed to execute the task) or a gain (generated by the termination of a product and its selling). As the firing of a transition produces the consumption of the tokens in its input places, it describes the end of the relevant tasks. The addition of their costs and gains (possibly multiplied by the value of the ingoing arcs) implies that the transition firing is also associated with a scalar that can be positive, negative or null. Formally, if the row-vector $P R$ represents the price for each place, the price relevant to one firing of a transition $i$ is 
$\sum_{j} P R_{j} \cdot W_{j, i}^{-}$. So, the balance-sheet on the period $[0, t]$ is

$$
P R \cdot W^{-} \cdot\left(\overline{\frac{x(t)}{y(t)}}\right)
$$

$$
\begin{gathered}
=c_{b a} \cdot \bar{\varkappa}+P R \cdot W_{o b s}^{-} \cdot \overline{y(t)} \text { where } \\
c_{b a}=\left(0_{1 \mathrm{x}\left|T R_{u n}\right|} 0_{1 \mathrm{x}\left|T R_{u n}\right|} \ldots 0_{1 \mathrm{x}\left|T R_{u n}\right|} c_{1 \mathrm{x}\left|T R_{u n}\right|}\right) \text { (ba: balance) with } \\
c_{1 \mathrm{x}\left|T R_{u n}\right|}=P R \cdot W_{u n}^{-}
\end{gathered}
$$

\section{Guaranteed time horizons}

Up to now, we have considered time sequences for a given step $\langle k\rangle$ and Timed Petri nets. In this section, the time Assumption $\mathcal{A S}-5$ is kept but Assumption $\mathcal{A S}-6$ saying that the dtes of observations are known is removed. In that case, a possibility is to assume that the horizon $h$ is taken sufficiently large such that the relations (8) describing the estimation problem presented in Section 3 do not limit the consistency of the untimed sequences and particularly the coherence between $\bar{y}^{<0>\rightarrow<k-1>}$ and $\bar{y}^{<0>\rightarrow<k>}$. However, the computations can be time costly and there is no guarantee that this value is sufficient for each considered estimation problem.

Therefore, the objective of Section 4 is to adapt the technique presented in Section 3 to the case where the dates of observations are unknown: the aim is to determine time horizons $h$ which does not limit the expressivity of every subsequence. The technique is based on the construction of untimed systems consistent with the Timed Petri net.

This part is organized as follows. The following Subsection 4.1 is the pivot point of the paper which makes the connection between the timed case and the untimed case. Different untimed systems in Subsection 4.2 are the supports of linear programming problems allowing the computation of the time horizons. Based on a relaxation over $\mathbb{R}$, the convergence of these problems to finite values is analyzed in Subsection 4.3. Finally, the introduction of the notion of Relaxed Structurally Boundedness in Subsection 4.4 makes the connections with standard definitions of SB and DSB Petri nets.

\subsection{Connection between the timed case and the untimed case}

We desire to find a time horizon corresponding to a possible time evolution of the Timed Petri net relevant to any sequence of the untimed Petri net for each step $\langle k\rangle$. The following result exploits a slow-down of the time behavior of the P-timed Petri net which is always possible as the token deaths are only determined by the consumption of the transition firings. 
Theorem 1 Let $c$ be an unitary row-vector with $|c|=\left|T R_{\text {un }}\right|$. The following optimization

$$
\nabla_{\max }^{<k>}=\max \left(c \cdot \bar{x}^{<k>}\right)
$$

where $x^{<k>}$ represents the unobservable sequence of the untimed Petri net for step $\langle k\rangle$, defines a guaranteed time horizon

$$
h_{g}=\nabla_{\max }^{<k>}+1
$$

for (8) if $\nabla_{\max }^{<k>}$ is finite $\left(\nabla_{\max }^{<k>} \in \mathbb{N}\right)$.

Proof. Let us now consider step $\langle k>$ where the untimed Petri net follows an arbitrary subsequence $x^{<k>} y^{<k>}$ starting from $M^{<k>}$. Formally, $M^{<k>}\left[x^{<k>} y^{<k>} \succ\right.$. At the beginning of step $\left\langle k>\right.$, vector $M^{<k>}$ is produced by the past evolution at step $<k-1>$ that is, $M^{<k-1>}\left[x^{<k-1>} y^{<k-1>} \succ\right.$ $M^{<k>}$. Let $q=\left\|\bar{x}^{<k>}\right\|_{1}$. If $q \neq 0$, the subsequence $x^{<k>} y^{<k>}$ can be developed under the form $x^{<k>}=x_{(1)} x_{(2)} \ldots x_{(i)} \cdots x_{(q)} y^{<k>}$ where $x_{(i)}$ represents the transition whose rank is $i \in\{1, \ldots, q\}$ in the subsequence in the untimed Petri net. Let $M^{<k, 1>}=M^{<k>}, M^{<k, i>}$ is the marking used at rank $i$ in step $\left\langle k>\right.$ and $M^{<k, i+1\rangle}$ is the expected marking produced by $x_{(i)}$. So, $M^{<k, 1>}\left[x_{(1)} \succ M^{<k, 2>}\left[x_{(2)} \succ M^{<k, 3>} \ldots M^{<k, i-1>}\left[x_{(i-1)} \succ\right.\right.\right.$ $M^{<k, i>} \ldots M^{<k, q>}\left[x_{(q)} \succ M^{<k, q+1>}\left[y^{<k>} \succ M^{<k+1>}\right.\right.$

Now, we desire that the associated P-time Petri net follows the same subsequence $x^{<k>} y^{<k>}$ : as the untimed Petri net satisfies $M^{<k>}\left[x^{<k>} y^{<k>} \succ\right.$ where $x^{<k>}$ and $y^{<k>}$ are subsequences whose count vectors are $\bar{x}^{<k>}$ and $\bar{y}^{<k>}$ respectively, we must find a time evolution which can always be satisfied by the relevant P-time Petri net. By construction (presented in Section 2.3), the last firing date of step $\left\langle k-1>\right.$ occurs at $t^{<k-1>}$. As the time durations are unitary, all the tokens produced by $x^{<k-1>} y^{<k-1>}$ are available at time $t^{<k-1>}+1$ and so, the marking $M^{<k>}$ is obtained at $t^{<k-1>}+1$.

We consider the following slow-down of the P-time Petri net where the rule is that a unique firing of a transition occurs at each time $\theta \in \mathbb{N}$.

As the marking $M^{<k>}$ is available at date $t^{<k-1>}+1$, the first unobservable transition $x_{(1)}$ can fire at the same date and modifies the marking and the new marking $M^{<k, 2>}$ is available at $t^{<k-1>}+2$ for sure. Formally, $M^{<k, 1>}\left[x_{(1)} \succ\right.$ $M^{<k, 2>}$ is guaranteed at time $t^{<k-1>}+2$ in the associated P-time Petri net. Similarly, for any $i \in\{1, \ldots, q\}$, each transition $x_{(i)}$ can fire at $t^{<k-1>}+i$ as this time is sufficient such that the marking modified by $x_{(1)} x_{(2)} \ldots x_{(i-1)}$ is available: $M^{<k>}\left[x_{(1)} x_{(2)} \ldots x_{(i)} \succ M^{<k, i+1>}\right.$ is guaranteed at time $t^{<k-1>}+i$ in the associated P-time Petri net. Therefore, the above rule allows that the P-timed Petri net can follow a time trajectory consistent with subsequence $x^{<k>}$ and the value $q=\sum_{i=1, \ldots,\left|T R_{u n}\right|} \overline{x_{i}}<k>=c . \bar{x}^{<k>}$ gives a time duration sufficiently large for all the firings relevant to $x^{<k>}$.

Finally, considering the last observation $y^{<k>}$ with $\left\|\bar{y}^{<k>}\right\|_{1}=1$, the optimization $\max \left(c . \bar{x}^{<k>}\right)+1$ for a given step $<k>$ provides the worst count vector which is a guaranteed horizon which cannot restrict the expressivity of every subsequence for step $\langle k\rangle$. 
In general, the guaranteed time horizon defined by (9) is not the smallest horizon and can be improved. We can consider a specific Petri net where the possible parallelism can reduce the necessary horizon to express the sequences. Another situation is the case of an unobservable input transition that is without input place: the firing can be immediate and occur without delay at time $t^{<k-1>}$. In that case, a reduction of the time horizon of one time unit can be made for each unobservable input transition.

\subsection{Untimed systems and linear programming problems}

Containing the unobservable subsequences and observable subsequences, each sequence whose firing at the initial marking is consistent with the observations must satisfy the enabling condition and the marking equation. Their application leads to system (3b) in Proposition 1 of [13] (also, (2) in Lemma 1 of [5], (5) in [7]). As demonstrated by the proof of this proposition (part Only if), it does not need the acyclicity assumption which is only necessary when the aim is to show that algebraic solutions satisfying this system corresponds to consistent sequences (part (If) in Proposition 1). The optimization of Theorem 1 where the objective is the determination of sufficient time horizons can be applied to this exact system or to a simplified form of this system. As the size of the exact system increases with the observations, we now explore the last case which can give systems where the dimensions only depend on the structure of the Petri net.

\subsubsection{Guaranteed time horizon for step $<1>$}

Let us consider the system at step $<1>$. Remember that $M^{<1>}$ is the initial marking $M^{\text {init }}$. Leading to the observation $y^{<1>}$, it exists a marking $M^{\prime}$ with

$$
M^{\prime}=M^{<1>}+W_{u n} \cdot \bar{x}^{<1>}
$$

satisfying $M^{\prime}\left[(y)^{<1>} \succ\right.$. So,

$$
M^{\prime}=M^{<1>}+W_{u n} \cdot \bar{x}^{<1>} \geq W_{o b s}^{-} \cdot \bar{y}^{<1>}
$$

or,

$$
-W_{u n} \cdot \bar{x}^{<1>} \leq b^{<1>}
$$

where $b^{<1>}=M^{<1>}-W_{o b s}^{-} \cdot \bar{y}^{<1>}$. Therefore, a guaranteed horizon for step $<1>$ is provided by the maximization

$$
\nabla_{\max }^{<1>}=\max \left(c . \bar{x}^{<1>}\right)
$$

with the constraints (12) and $\bar{x}^{<1>} \geq 0$.

Example 3 continued.

This example allows to illustrate the computation of a guaranteed horizon for step $<1>$. Consider the Petri net of Fig. 2 and the relevant untimed 
system (12). We take $M^{<1>}=\left(\begin{array}{l}1 \\ 0\end{array}\right)$ and the observation is $y_{2}$ at step $<1>$ : so, $\bar{y}_{2}^{<1>}=1$. As $M_{2}^{<1>}=0$, the analysis of the Petri net shows that the firing of $x_{1}$ is necessary. So, $M^{<1>}\left[x_{1} \succ\left(\begin{array}{l}0 \\ 1\end{array}\right)\left[y_{2} \succ\left(\begin{array}{l}0 \\ 0\end{array}\right)\right.\right.$ and a possible time trajectory which can describe the evolution is \begin{tabular}{r|r|rr}
$\theta$ & 0 & 1 & 2 \\
\hline$\frac{x_{1}(\theta)}{y_{2}(\theta)}$ & 0 & 1 & 1 \\
0 & 0 & 1
\end{tabular}. . So, we can take $t^{<1>}=2$ and the time horizon $t^{<1>}-t^{<0>}=2$ or greater values are sufficient for describe the sequence of step $<1>$. Let us confirm this result.

$$
\text { As } W_{u n}=\left(\begin{array}{r}
-1 \\
1
\end{array}\right), W_{o b s}^{-}=\left(\begin{array}{ll}
0 & 0 \\
0 & 1
\end{array}\right) \text { and } W_{o b s}=\left(\begin{array}{rr}
1 & 0 \\
0 & -1
\end{array}\right) \text {, inequality }
$$
$\left(\begin{array}{r}1 \\ -1\end{array}\right) \cdot \bar{x}^{<1>} \leq\left(\begin{array}{r}M_{1}^{<1>} \\ M_{2}^{<1>}-y_{2}^{<1>}\end{array}\right)=\left(\begin{array}{r}1 \\ -1\end{array}\right)$ which gives $\bar{x}^{<1>}=1=\nabla_{\max }^{<1>}$ and $h_{g}=\nabla_{\max }^{<k>}+1=2$ which is coherent with the above results.

\subsubsection{Guaranteed time horizon for the step horizon $\langle 1\rangle \ldots<k>$}

We now consider a step horizon going from $\langle 1>$ to $\langle k>$ with $k \geq 2$. Let

$$
\nabla_{\max }^{<0>\rightarrow<k>}=\max \left(c \cdot \bar{x}^{<0>\rightarrow<k>}\right) .
$$

As $M^{<k>}=M^{<1>}+W_{u n} \cdot \bar{x}^{<0>\rightarrow<k-1>}+W_{\text {obs }} \cdot \bar{y}^{<0>\rightarrow<k-1>}$, the inequality allowing the observation $y^{<k>}$ for the marking $M^{\prime}=M^{<k>}+W_{u n} \cdot \bar{x}^{<k>}$ can be rewritten as follows

$$
-W_{u n} \cdot \bar{x}^{<0>\rightarrow<k>} \leq b^{<k>}
$$

where $b^{<k>}=M^{<1>}+W_{o b s} \cdot \bar{y}^{<0>\rightarrow<k-1>}-W_{o b s}^{-} \cdot \bar{y}^{<k>}$ for $k \geq 2$.

Therefore, the integer linear programming problem

$$
\begin{gathered}
\nabla_{\max }^{<0><k>}=\max \left(c . \bar{x}^{<0>\rightarrow<k>}\right) \\
-W_{u n} . \bar{x}^{<0>\rightarrow<k>} \leq b^{<k>}
\end{gathered}
$$

with

$$
\begin{gathered}
\bar{x}^{<0>\rightarrow<k>} \geq 0, \\
b^{<k>}=M^{<1>}+W_{o b s} \cdot \bar{y}^{<0>\rightarrow<k-1>}-W_{o b s}^{-} \cdot \bar{y}^{<k>}
\end{gathered}
$$

computes a guaranteed time horizon for the step horizon going from $<1>$ to $<k>$ for $k \geq 2$ if the computed value is finite.

4.2.3 Guaranteed time horizon for a step $<k>$ with $k \geq 2$

Let us focus on a guaranteed horizon relevant to a step $\langle k>$ with $k \geq 2$. From (15), we simply obtain $-W_{u n} \cdot \bar{x}^{<0>\rightarrow<k-1>}-W_{u n} \cdot \bar{x}^{<k>} \leq b^{<k>}$ 
Moreover, the condition $M^{<k>} \geq 0$ with $M^{<k>}=M^{<1>}+W_{u n} \cdot \bar{x}^{<0>\rightarrow<k-1>}+$ $W_{\text {obs }} \cdot \bar{y}^{<0>\rightarrow<k-1>}$ can also be used. We obtain

$$
\begin{gathered}
\left(\begin{array}{cc}
-W_{u n} & 0 \\
-W_{u n}-W_{u n}
\end{array}\right) \cdot\left(\begin{array}{c}
\bar{x}^{<0>\rightarrow<k-1>} \\
\bar{x}^{<k>}
\end{array}\right) \leq \\
\left(\begin{array}{c}
M^{<1>}+W_{o b s} \cdot \bar{y}^{<0>\rightarrow<k-1>} \\
b^{<k>}
\end{array}\right)
\end{gathered}
$$

Therefore, a guaranteed horizon for step $\langle k\rangle$ is provided by the maximization

$$
\nabla_{\max }^{<k>}=\max \left(c . \bar{x}^{<k>}\right)
$$

with the constraints $(17), \bar{x}^{<k>} \geq 0$ and $\bar{x}^{<0>\rightarrow<k-1>} \geq 0$.

The dimensions of (12), (15) and (17) are constant and only depend on the parameters of the Petri net and not the sequences: the sizes of the systems are $|P| \mathrm{x}\left|T R_{u n}\right|,|P| \mathrm{x}\left|T R_{u n}\right|$ and $2 .|P| \mathrm{x} 2 .\left|T R_{u n}\right|$ respectively.

Example 3 continued.

This example allows to illustrate the computation of a guaranteed horizon for step $\langle k\rangle$. Consider the Petri net of Fig. 2 and the relevant untimed system (17). We take $M^{<1>}=\left(\begin{array}{l}1 \\ 0\end{array}\right)$ and the sequence of observations is $y_{2} y_{1} y_{2}$. So, $y^{<1>}=y_{2}, y^{<2>}=y_{1}$ and $y^{<3>}=y_{2}$. The analysis of the Petri net shows that $M^{<1>}\left[x_{1} \succ\left(\begin{array}{l}0 \\ 1\end{array}\right)\left[y_{2} \succ\left(\begin{array}{l}0 \\ 0\end{array}\right)\left[y_{1} \succ\left(\begin{array}{l}1 \\ 0\end{array}\right)\left[x_{1} \succ\left(\begin{array}{l}0 \\ 1\end{array}\right)\left[y_{2} \succ\left(\begin{array}{l}0 \\ 0\end{array}\right)\right.\right.\right.\right.\right.$ and $x^{<1>}=x_{1}, x^{<2>}=\varepsilon$ and $x^{<3>}=x_{1}$. A possible time trajectory which can describe the evolution is

$$
\begin{array}{|r|llllll|}
\hline \theta & 0 & 1 & 2 & 3 & 4 & 5 \\
\hline \overline{x_{1}(\theta)} & 0 & 1 & 1 & 1 & 2 & 2 \\
\hline y_{1}(\theta) & 0 & 0 & 0 & 1 & 1 & 1 \\
y_{2}(\theta) & 0 & 0 & 1 & 1 & 1 & 2 \\
\hline
\end{array}
$$

For steps $<1>,<2>$ and $<3>$, we take $t^{<1>}=2, t^{<2>}=3$ and $t^{<3>}=$ 5 corresponding to the observations $y_{2}, y_{1}$ and $y_{2}$, respectively. The horizons $h^{<1>}=t^{<1>}-t^{<0>}=2, h^{<2>}=t^{<2>}-t^{<1>}=1$ and $h^{<3>}=t^{<3>}-t^{<2>}=2$ are sufficient to express the relevant trajectories. Let us verify these results.

Inequality (15) at iteration $<3>$ becomes

$\left(\begin{array}{r}1 \\ -1\end{array}\right) \cdot \bar{x}^{<0>\rightarrow<3>} \leq\left(\begin{array}{r}M_{1}^{<1>}+\bar{y}_{1}^{<0>\rightarrow<2>} \\ M_{2}^{<1>}-\bar{y}_{2}^{<0>\rightarrow<2>}-\bar{y}_{2}^{<3>}\end{array}\right)=\left(\begin{array}{r}2 \\ -2\end{array}\right)$ which gives
$\bar{x}^{<0>\rightarrow<3>}=2=\nabla_{\max }^{<0>\rightarrow<3>} ;$

Inequality (17) at $<3>$ becomes

$$
\left(\begin{array}{rr}
1 & 0 \\
-1 & 0 \\
1 & 1 \\
-1 & -1
\end{array}\right) \cdot\left(\begin{array}{r}
\bar{x}^{<0>\rightarrow<2>} \\
\bar{x}^{<3>}
\end{array}\right) \leq\left(\begin{array}{r}
M_{1}^{<1>}+\bar{y}_{1}^{<0>\rightarrow<2>} \\
M_{2}^{<1>}-\bar{y}_{2}^{<0>\rightarrow<2>} \\
M_{1}^{<1>}+\bar{y}_{1}^{<0>\rightarrow<2>} \\
M_{2}^{<1>}-\bar{y}_{2}^{<0>\rightarrow<2>}-\bar{y}_{2}^{<3>}
\end{array}\right)=\left(\begin{array}{r}
2 \\
-1 \\
2 \\
-2
\end{array}\right)
$$

and its resolution yields $0 \leq \bar{x}^{<3>} \leq 1$. So, $\nabla_{\max }^{<3>}=\max \left(c . \bar{x}^{<3>}\right)=1$ and $h_{g}^{<3>}=\nabla_{\max }^{<3>}+1=2$ for step $<3>$ which is coherent with the above result $h^{\circ}<>=2$. 
As these systems provide guaranteed majorants with the condition that the optimization converges to a finite solution, we below analyze the conditions such that the problems (16) and (18) can give a finite solution. We define the boundedness of a linear programming problem as the possibility that the optimal value of the criterion is upper bounded. To facilitate the analysis, we consider the boundedness of the problem (16) which implies the boundedness of the problem (18) as (15) is the last row of (17). Moreover, a relaxation over $\mathbb{R}$ allows to exploit useful results of linear programming and also the efficient softwares of linear programming. Note that the relaxation gives a value equal to or greater than the value obtained by the direct application of softwares of integer linear programming.

\subsection{Convergence of problem (16) over $\mathbb{R}$}

The objective is now to know if $\nabla_{\max }^{<0>\rightarrow<k>}$ is finite otherwise the approach cannot be applied in all cases. Exploiting the duality, the following result suggests that the maximization (9) provides a finite value when the unobservable subnet presents a specific characteristic which can be checked once in the estimation problem.

Theorem 2 The maximization (9) over $\mathbb{R}$ is upper bounded if there is $z \geq 0$ over $\mathbb{R}$ such that $z . W_{\text {un }} \leq-c$ with $c$ unitary.

$$
\text { Moreover, } c . \bar{x}^{<0>\rightarrow<k>} \leq z \cdot b^{<k>}
$$

\section{Proof.}

We search $\max ($ c. $x)$ such that $A . x \leq b$ with $x=\bar{x}^{<0>\rightarrow<k>} \geq 0$ over the integers, $A=-W_{u n}$ and $b=b^{<k>}$.

If we relax the problem over $\mathbb{R}$, the application of the duality theorem implies

$\min (z . b)$ such that $z \cdot A \geq c$ with $z \geq 0$ over $\mathbb{R}$ or

$z . W_{u n} \leq-c$ with $z \geq 0$ over $\mathbb{R}$ and $c$ unitary with the notations of this paper. The theorem 4.5 in [8] shows that any possible finite solution $z$ implies the existence of $x$ satisfying $A . x \leq b$ and provides a majorant $z . b$ to $c . x$, or $c . x \leq z . b$ (indeed, $A . x \leq b$ and $z . A \geq c$ implies $z . b \geq z . A . x \geq c . x$ )

With the notations of this paper, we obtain $c \cdot \bar{x}^{<0>\rightarrow<k>} \leq z . b^{<k>}$ and any solution $z \geq 0$ over $\mathbb{R}$ provides a majorant of the criterion for the problem relaxed over $\mathbb{R}$ and ad fortiori to the initial problem which is more restrictive as defined over $\mathbb{N}$. Note that the integer solution $\bar{x}^{<0\rangle \rightarrow\langle k\rangle}$ is also finite as the obtained value $c \cdot \bar{x}^{<0>\rightarrow<k>}$ is finite and $c$ unitary does not contain null component.

Note that conversely any possible finite solution $\bar{x}^{<0>\rightarrow<k>}$ provides a minorant $c . x$ to $z . b^{<k>}$.

Corollary 1 If the maximization (9) over $\mathbb{R}$ is not upper bounded, then we cannot find $z \geq 0$ over $\mathbb{R}$ such that $z$. $W_{\text {un }} \leq-c$. 


\section{Proof.}

This corollary is directly deduced from the negation of the above proposition. Another reasoning is as follows.

If the maximization (9) over $\mathbb{R}$ is not upper bounded, then $\max (c . x)=+\infty$ which implies an impossibility in the dual problem as any solution to $z \cdot A \geq c$ must also satisfy $z . b \geq c . x=+\infty$ which is impossible over $\mathbb{R}$ as $z$ and $b$ are finite.

Property 1 The case $\min (z . b)=-\infty$ is not possible in the estimation problem.

\section{Proof.}

Assume that $\min (z . b)=-\infty$. Therefore, in the primal problem, any solution to $A . x \leq b$ must also satisfy $c . x \leq z . b \leq-\infty$ which is impossible as $c$ unitary and $x \geq 0$. Note that the practical context of the paper implies that the Petri net always follows a sequence: $c . x \geq 0$.

The duality theorem implies over $\mathbb{R}$

$$
\max \left(c \cdot \bar{x}^{<0>\rightarrow<k>}\right)=\min \left(z \cdot b^{<k>}\right)
$$

The condition of existence of $z \geq 0$ in the Corollary and Theorem 2 can be checked with the following linear programming problem

$$
\begin{gathered}
\min \left(c^{\prime} \cdot z^{T}\right) \\
W_{u n}^{t} \cdot z^{T} \leq-c^{T} \\
z^{T} \geq 0
\end{gathered}
$$

with $c^{\prime}$ a unitary row-vector and $\left|c^{\prime}\right|=|z|$. The minimization avoids the problem of boundedness and an unfeasible problem means that there is no $z$ satisfying the constraints.

\subsection{Discussion}

Let us make the connections with the definitions of SB and DSB Petri nets which are close. The following definition modifies the condition in Theorem 2 by replacing $z \cdot W_{u n} \leq-c$ by $z \cdot W_{u n}<0$.

Definition 4 The unobservable induced Petri net is Relaxed Structurally Bounded (RSB) if and only if there exists a non-negative vector $z$ over $\mathbb{R}$ such that $z \cdot W_{u n}<0$. Formally,

$$
\exists z \geq 0 \text { such that } z \cdot W_{u n}<0 \text { with } z \text { over } \mathbb{R}
$$

So, the last definition considers the unobservable induced subnet and not the complete Petri net as in definitions of SB and DSB Petri nets. We consider the real numbers while the definitions of SB and DSB Petri nets are over the integers. Moreover, the inequalities are different as some components of $z$ in the proposed definition can be null $(z \geq 0)$. We present the following result which makes the connection with Theorem 2 . 
Property 2 The maximization in (16) is upper bounded if the unobservable subnet is RSB.

Proof. From $-W_{u n} \cdot \bar{x}^{<0>\rightarrow<k>} \leq b^{<k>}$, we can write

$-z \cdot W_{u n} \cdot \bar{x}^{<0>\rightarrow<k>} \leq z \cdot b^{<k>}$ as $z \geq 0$ does not modify the sign.

Moreover, $-z . W_{u n}>0$ by definition: we can always make a multiplication by a positive scalar $\lambda$ such that $-\lambda . z . W_{u n} \geq c$ with $c=\left(\begin{array}{lll}1 & 1 \ldots 1\end{array}\right)$ or $-z^{\prime} \cdot W_{u n} \geq c$ with $z^{\prime}=\lambda . z$, the inequality becomes $c . \bar{x}^{<0>\rightarrow<k>} \leq$ $-z^{\prime} \cdot W_{u n} \cdot \bar{x}^{<0>\rightarrow<k>} \leq z^{\prime} \cdot b^{<k>}$ with $c$ unitary. As the right-hand term $z^{\prime} \cdot b^{<k>}$ is a (finite) majorant of the left-hand term for any evolution of the system, we can deduce that $c . \bar{x}^{<0>\rightarrow<k>}$ is upper bounded and is always finite.

\section{Simple example with unobservable circuit (example 4)}

Let us consider the Untimed Labelled Petri Net (LPN) of Fig. 3 which contains an unobservable circuit. The simplicity of this Petri net allows to present easily the main concepts of the approach.

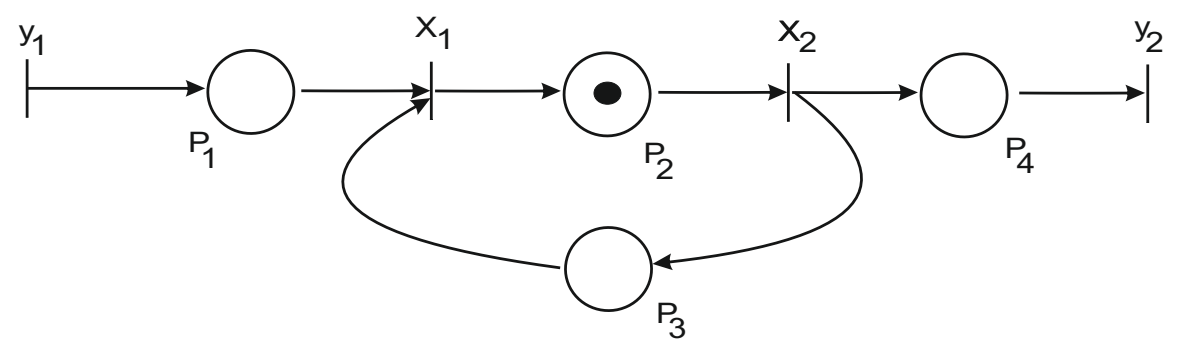

Fig. 3 A simple Petri with an unobservable circuit (example 4)

Let $T R_{u n}=\left\{x_{1}, x_{2}\right\}$ and $T R_{o b s}=\left\{y_{1}, y_{2}\right\}$. The alphabet $A L$ is $\{a, b\}$. Label $a$ is associated with the transition $x_{1}\left(\Omega_{a}=\left\{x_{1}\right\}\right)$, and label $b$ is associated with the transition $x_{2}\left(\Omega_{b}=\left\{x_{2}\right\}\right.$. Hence, $n=\left|T R_{u n}\right|=2, n^{\prime}=\left|T R_{o b s}\right|=2$, $n^{\prime \prime}=|A L|=2, m=|P|=4$.

The incidence matrices $W_{u n}, W_{o b s}, W_{o b s}^{-}$and the initial marking are as follows: $W_{u n}=\left(\begin{array}{rr}-1 & 0 \\ 1 & -1 \\ -1 & 1 \\ 0 & 1\end{array}\right), W_{o b s}=\left(\begin{array}{rr}1 & 0 \\ 0 & 0 \\ 0 & 0 \\ 0 & -1\end{array}\right), W_{o b s}^{-}=\left(\begin{array}{ll}0 & 0 \\ 0 & 0 \\ 0 & 0 \\ 0 & 1\end{array}\right)$ and $M^{<1>}=$ $\left(\begin{array}{llll}0 & 1 & 0 & 0\end{array}\right)^{T}$. The unobservable subnet is RSB as $\exists z=\left(\begin{array}{llll}3 & 3 & 1 & 1\end{array}\right)>0$ such that $z . W_{u n}=(-1-1)<0$. Therefore, the proposed approach can be applied in all situations.

Subsequence to be estimated. We consider the following possible trajectory followed by the Petri net which is assumed to be unknown. Starting from the initial marking $M^{<1>}=\left(\begin{array}{llll}0 & 1 & 0 & 0\end{array}\right)^{T}$, an obtained sequence is 
$x_{2} y_{1} x_{1} y_{2} x_{2} y_{2} y_{1}$ which contains the sequence of observable transitions $y_{1} y_{2} y_{2} y_{1}$ relevant to the sequence of labels $a b b a$ and corresponding to the count vectors $\bar{y}^{<k>}$ given in the table 1 for each step $\langle k>$ which also contains the subsequence of unobservable transitions and $\bar{x}^{<k>}$. In the sequel, the objective is to estimate these unknown subsequences and to compute some criteria.

Table 1 Simulation: observations and unknown data to be estimated

\begin{tabular}{|c|cccc|}
\hline$<k>$ & 1 & 2 & 3 & 4 \\
\hline Observation & $y_{1}$ & $y_{2}$ & $y_{2}$ & $y_{1}$ \\
$\bar{y}^{<k>}$ & $\left(\begin{array}{l}1 \\
0\end{array}\right)$ & $\left(\begin{array}{l}0 \\
1\end{array}\right)$ & $\left(\begin{array}{l}0 \\
1\end{array}\right)$ & $\left(\begin{array}{l}1 \\
0\end{array}\right)$ \\
Subsequence & $x_{2}$ & $x_{1}$ & $x_{2}$ & $\varepsilon$ \\
$\bar{x}^{<k>}$ & $\left(\begin{array}{l}0 \\
1\end{array}\right)$ & $\left(\begin{array}{l}1 \\
0\end{array}\right)$ & $\left(\begin{array}{l}0 \\
1\end{array}\right)$ & $\left(\begin{array}{l}0 \\
0\end{array}\right)$ \\
\hline
\end{tabular}

\section{Phase 1: Determination of a guaranteed horizon}

Let us check that the computed time horizons are finite. System (15)

$-W_{u n} \cdot \bar{x}^{<1>\rightarrow<i>} \leq M^{<1>}+W_{o b s} \cdot \bar{y}^{<1>\rightarrow<i-1>}-W_{o b s}^{-} \cdot \bar{y}^{<i>}$ for $i \geq 2$ becomes:

$$
\left(\begin{array}{rr}
1 & 0 \\
-1 & 1 \\
1 & -1 \\
0 & -1
\end{array}\right) . \bar{x}^{<0>\rightarrow<k>} \leq\left(\begin{array}{r}
\bar{y}_{1}^{<0>\rightarrow<k-1>} \\
1 \\
0 \\
-\bar{y}_{2}^{<0>\rightarrow<k>}
\end{array}\right) \text {. The maximization of }
$$

with $c$ unitary provides the time horizon $\nabla_{\max }^{<0>} \rightarrow\langle k>$ for each step $\langle k\rangle$. The obtained values can be verified by the following algebraic resolution. As the system is sup-monotone (the system of linear inequalities $A . x \leq b$ is infmonotone (respectively, sup-monotone ) if each row of matrix $A$ has one strictly negative (respectively positive) element at most [9]), a greatest solution exists and is $\overline{x_{1}^{+}}=\bar{y}_{1}^{<0>\rightarrow<k-1>}$ and $\overline{x_{2}^{+}}=1+\overline{x_{1}}<0>\rightarrow<k-1>$ under the condition of existence $\bar{y}_{2}^{<0>\rightarrow<k>} \leq \overline{x_{2}^{+}}$(this relation is always satisfied as it is deduced from a consistent model describing the considered live Petri net. In addition, all the considered observations coming from this Petri net are exact. Otherwise, other estimation approaches must be used [10]). The estimated time horizon $\nabla \underset{\max }{<0><k>}$ is the sum of the firing numbers of the unobservable transitions computed with the above relation, that is, $\overline{x_{1}^{+}}+\overline{x_{2}^{+}}=1+2 . \bar{y}_{1}^{<0>\rightarrow<k-1>}$ which is always finite.

A candidate horizon $\nabla_{\max }^{<k>}$ (which is used in the estimation of subsequences below) is given by the maximization in (18) based on system (17). The first three lines of the following table 2 represent the known data while the last four lines are the computed results.

Therefore, the obtained values of $\nabla_{\max }^{<0>} \rightarrow\left\langle k>\right.$ and $\nabla_{\max }^{<k>}$ are finite and the proposed approach can be applied. We can check that $c \cdot \bar{x}<k>\leq \nabla_{\max }^{<k>} \leq$ $\nabla_{\max }^{<0>} \rightarrow<k>$ for each iteration $\left\langle k>\right.$ where $\bar{x}^{<k>}$ is the result of the simulation in Table 1. 
Table 2 Greatest estimate and computed $\nabla$

\begin{tabular}{|c|c|c|c|c|}
\hline$\langle k\rangle$ & 1 & 2 & 3 & 4 \\
\hline $\bar{y}<0>\rightarrow<k-1>$ & 0 & 1 & 1 & \\
\hline $\bar{x}<k>$ & & & & \\
\hline$x_{1}^{+}$ & 0 & 1 & 1 & 1 \\
\hline$x_{2}^{+}$ & 1 & 2 & 2 & 2 \\
\hline$\nabla_{\max }^{<0>\rightarrow<k>}$ & 1 & 3 & 3 & 3 \\
\hline$\nabla_{\max }^{<}<k>$ & 1 & 3 & 2 & 0 \\
\hline
\end{tabular}

\section{Phase 2: Estimation of subsequences}

- Let us consider iteration $<4>$ which leads to the simplest calculations: we have $h_{g}=\nabla_{\max }^{<4>}+1=1$ for $\langle 4>$ as there is a unique observation.

As $\nabla_{\max }^{<4>}=0$, we can deduce that $\bar{x}^{<k>}=0$ and $\left(\overline{x\left(t-h_{g}\right)}\right)=(\overline{x(t)})$. Let us verify this result. We have $n=2 .\left|T R_{u n}\right|$. The dimensions of matrices $\mathbf{A}_{1}$, $\mathbf{B}_{1}, \mathbf{C}_{1}$ and column vector $\mathbf{b}_{1}=\mathbf{C}_{1}-\mathbf{B}_{1} \cdot \bar{\gamma}$ are respectively $\left(|P| \times 2 .\left|T R_{u n}\right|\right)$, $\left(|P| \times 2 .\left|T R_{o b s}\right|\right),(|P| \times 1)$ and $(|P| \times 1) \cdot \bar{\gamma}=$

$$
\begin{aligned}
& \left(\left(\bar{y}^{<0>\rightarrow<k-1>}\right)^{T}\left(\bar{y}^{<0>\rightarrow<k>}\right)^{T}\right)^{T}=\left(\begin{array}{llll}
1 & 2 & 2 & 2
\end{array}\right)^{T} \text { and } \\
& \bar{\varkappa}=\left((\overline{x(t-1)})^{T}(\overline{x(t)})^{T}\right)^{T} \text {. }
\end{aligned}
$$

$$
\mathbf{A}_{1} \cdot \bar{\varkappa} \leq \mathbf{C}_{1}-\mathbf{B}_{1} \cdot \bar{\gamma}
$$

where $\mathbf{A}_{1}=\left(-W_{u n}^{+} W_{u n}^{-}\right), \mathbf{B}_{1}=\left(-W_{o b s}^{+} W_{o b s}^{-}\right)$and $\mathbf{C}_{1}=\left(M^{<1>}\right)$.

So,

$$
\begin{aligned}
& \left(-W_{\text {un }}^{+} W_{\text {un }}^{-}\right) \cdot(\overline{\overline{x(t-1)}}) \leq M^{<1>}-\left(-W_{\text {obs }}^{+} W_{o b s}^{-}\right) \cdot(\overline{\overline{x(t)} \overline{y(t)}}) \text { or } \\
& \left(\begin{array}{rrrr}
0 & 0 & 1 & 0 \\
-1 & 0 & 0 & 1 \\
0 & -1 & 1 & 0 \\
0 & -1 & 0 & 0
\end{array}\right) \cdot\left(\overline{x\left(\frac{t-1)}{x(t)}\right.}\right) \leq\left(\begin{array}{l}
0 \\
1 \\
0 \\
0
\end{array}\right)-\left(\begin{array}{rrrr}
-1 & 0 & 0 & 0 \\
0 & 0 & 0 & 0 \\
0 & 0 & 0 & 0 \\
0 & 0 & 0 & 1
\end{array}\right) \cdot\left(\overline{y\left(\frac{t-1)}{\overline{y(t)}}\right)}\right.
\end{aligned}
$$

For $\overline{y(t-1)}=\left(\begin{array}{l}1 \\ 2\end{array}\right)$ and $\overline{y(t)}=\left(\begin{array}{l}2 \\ 2\end{array}\right)$ at iteration $<4>$, a simple resolution gives a unique solution with $\left(\overline{x\left(t-h_{g}\right)}\right)=(\overline{x(t)})=\left(\begin{array}{l}1 \\ 2\end{array}\right)$ which corresponds to one firing of $x_{1}$ and two firing of $x_{2}$ from iteration $\langle 1\rangle$ to $<4>$. The equality $\left(\overline{x\left(t-h_{g}\right)}\right)=(\overline{x(t)})$ expresses that there is no new firing of unobservable transitions for iteration $\langle 4\rangle$, that is $\bar{x}^{<k\rangle}=0$.

- Let us consider iteration $\langle 3\rangle$. We have $h_{g}=\nabla_{\max }^{<3>}+1=3$. The dimension of vector $\bar{\varkappa}$ is denoted by $n=4$. $\left|T R_{u n}\right|$ while the dimension of vector $\bar{\gamma}$ is $4 .\left|T R_{\text {obs }}\right|$. The dimensions of matrices $\mathbf{A}_{1}, \mathbf{B}_{1}, \mathbf{C}_{1}$ and column vector $\mathbf{b}_{1}=\mathbf{C}_{1}-\mathbf{B}_{1} \cdot \bar{\gamma}$ are respectively $(3 .|P| \mathrm{x} n),\left(3 .|P| \mathrm{x} 4 .\left|T R_{o b s}\right|\right),(3 .|P|$ $\mathrm{x} 1)$ and $(3 .|P| \times 1)$. 


$$
\bar{\gamma}=\left(\left(\bar{y}^{<1>\rightarrow<2>}\right)^{T}\left(\bar{y}^{<1>\rightarrow<2>}\right)^{T} \quad\left(\bar{y}^{<1>\rightarrow<2>}\right)^{T}\left(\bar{y}^{<1>\rightarrow<3>}\right)^{T}\right)^{T}
$$

with $\bar{y}^{<1>\rightarrow<2>}=\left(\begin{array}{l}1 \\ 1\end{array}\right)$ and $\bar{y}^{<1>\rightarrow<3>}=\left(\begin{array}{l}1 \\ 2\end{array}\right)$.

$$
\bar{\varkappa}=\left((\overline{x(t-3)})^{T}(\overline{x(t-2)})^{T} \quad(\overline{x(t-1)})^{T}(\overline{x(t)})^{T}\right)^{T},
$$

$$
\mathbf{A}_{1} \cdot \bar{\varkappa} \leq \mathbf{C}_{1}-\mathbf{B}_{1} \cdot \bar{\gamma}
$$

where

$$
\begin{aligned}
\mathbf{A}_{1} & =\left(\begin{array}{cccc}
-W_{u n}^{+} & W_{u n}^{-} & 0 & 0 \\
0 & -W_{u n}^{+} & W_{u n}^{-} & 0 \\
0 & 0 & -W_{u n}^{+} & W_{u n}^{-}
\end{array}\right), \\
\mathbf{B}_{1} & =\left(\begin{array}{cccc}
-W_{o b s}^{+} & W_{o b s}^{-} & 0 & 0 \\
0 & -W_{o b s}^{+} & W_{o b s}^{-} & 0 \\
0 & 0 & -W_{o b s}^{+} & W_{o b s}^{-}
\end{array}\right) \text {and } \mathbf{C}_{1}=\left(\begin{array}{c}
M^{<1>} \\
M^{<1>} \\
M^{<1>}
\end{array}\right) .
\end{aligned}
$$

The minimization and maximization $c . \overline{x(t)}$ with $c$ unitary gives $(\overline{x(t)})=$ $\left(\begin{array}{lll}1 & 2\end{array}\right)^{T}$. The minimization $c_{\text {diff }} \cdot \bar{\varkappa}$ gives $\bar{\varkappa}=\left(\begin{array}{lllllll}1 & 2 & 1 & 2 & 1 & 2 & 1\end{array}\right)^{T}$ which corresponds to no firing (no variation of the count number vector which is always $\left(\begin{array}{ll}1 & 2\end{array}\right)^{T}$ ) and the maximization gives

$\bar{\varkappa}=\left(\begin{array}{llllllll}0 & 1 & 1 & 1 & 1 & 2 & 1 & 2\end{array}\right)^{T}$ which describes the subsequence $x_{1} x_{2}$ for the iteration $<3>$ (change $(0,1) \rightarrow(1,1)$ in $\left(\begin{array}{llll}0 & 1 & 1 & 1\end{array}\right)^{T}$ gives $x_{1},(1,1) \rightarrow(1,2)$ gives $x_{2}$ and $(1,2) \rightarrow(1,2)$ gives $\left.\varepsilon\right)$. So, for step $<3>$, the estimate $x_{1} x_{2}$ includes the subsequence of the simulation $x_{2}$ which includes $\varepsilon$. The same remark holds for the other steps.

In table 3 , the first part represents the simulation of the Petri net where $\overline{y(t)}$ and $\bar{x}^{<k>}$ for $\langle k>$ are given. Considering the minimization and the maximization of the criterion $c . \bar{\varkappa}$ with $c=c_{\text {diff }}$ for each step $<k>$, the second part gives the estimated subsequence (deduced from the trajectory $\bar{\varkappa})$ and the relevant vectors $\bar{x}^{<k>}$ (deduced from $\bar{x}^{<k>}=\overline{x(t)}-\overline{x(t-h)}$ ) which are denoted $\left[\overline{x_{u n}(t)}\right]^{-}$and $\left[\overline{x_{u n}(t)}\right]^{+}$respectively. The tests show that $\left[\bar{x}^{<k>}\right]^{-} \leq \bar{x}^{<k>} \leq\left[\bar{x}^{<k>}\right]^{+}$where $\bar{x}^{<k>}$ is the count vector of the unknown subsequence to be estimated. The analysis of the results shows that the unknown subsequence to be estimated is included by the estimated subsequence for $\max \left(c_{\text {diff }} \cdot \bar{\varkappa}\right)$ and includes the estimated subsequence for $\min \left(c_{\text {diff } f} \cdot \bar{\varkappa}\right)$. The estimation is exact for step $\langle 4\rangle$. Note that the values of the criterion are always lower than or equal to $\nabla_{\max }^{<k>}$ computed in Table 2.

Taking $c=c_{b a}$, the last part presents the minimum and maximum of the balance-sheet on the period $[0, t]$ for $P R=(-1020-158)$ : places $p_{1}$ and $p_{3}$ have a cost 10 and 15 while places $p_{2}$ and $p_{4}$ have a gain 20 and 8 respectively.

\section{Comparison with some approaches}

In [13], the diagnosis approach of partially observed LPN is based on the resolution of some Integer Linear Programming (ILP) problems for each observed transition. A fault expressed by an unobservable transition firing is detected 
Table 3 Estimation of subsequences and balance-sheets by step

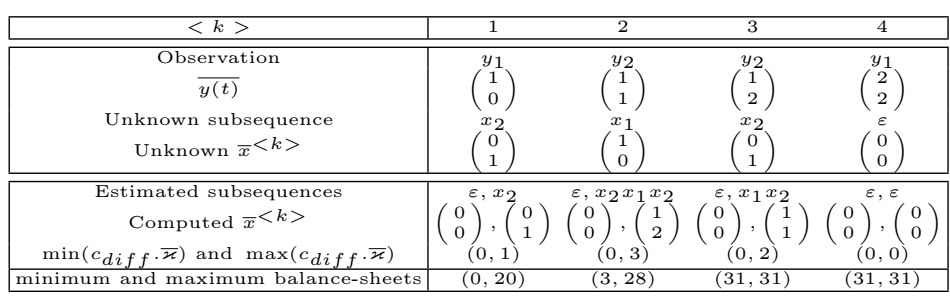

if it is included in a subset of unobservable sequences coherent with the observation verifying an ILP problem. The authors proposed a diagnosis approach based on the resolution of at most $F+2$ ILP problems where $F$ is the number of faults that may occur in the system. The effectiveness was indicated in the particular case where the unobservable subnet of a bounded LPN was an acyclic "state machine" where the incidence matrix of the unobservable subnet is totally unimodular. In that case, the solutions of the relaxed problem are integers and the resolution presents a polynomial time. The drawback of approaches [13] and also [7] is that the receding horizon could not be increased infinitely as it rises with the cardinality of the observed word and the relevant execution time. In this paper, the technique based on a sliding window avoids this limitation.

In the literature, the case of cyclic unobservable induced subnet has been considered by only few studies as [19] [7] [5]. The study [19] gives an example presenting a large initial marking where an approach based on a "fluidification" can be used when discrete approaches are not applicable in practice. Using the convexity property, the diagnosis approach is based on the computation of a set of vertices of a polytope for each observed transition. The firing vectors of unobservable sequences consistent with an observed transition is the convex hull of the union of all the vertices thus obtained. The drawback of this approach is that the time to compute the diagnosis status at a given observation would increase when the number of vertices at the previous observation rose and the determination of the complexity is a perspective.

Analyzing an algebraic description of Petri nets close to [13], the paper [5] focuses on the diagnosability of a fault which is useful during the design phase. Considering any sequence generated by the system that ends in a failure event, diagnosability implies that along every continuation of this sequence it is possible to detect the occurrence of the fault with a finite length of the continuation and a problem is the existence of an upper bound for the continuation of the sequence. The paper [5] focuses on a relevant quantitative bound for bounded Petri nets and checks if the designed system fulfills an algebraic feasibility problem in terms of number of events needed to detect the faults. Treated with ILP, a practical question is the possibility to specify a majorant for the number of events of any sequence that ends in the failure and an upper bound depending of the initial marking and the T-invariants is presented. 
Section 4 of this paper presents some similarities, as the goal is about a majorant of a sequence length and the case where only a single transition is enabled under each reachable marking (the concurrence cannot be exploited) is used in the establishment of the majorant. However, the objective is different as we focus on the on-line procedure and not on the diagnosability. Our aim is to determine a guaranteed time horizon which does not limit the expressivity of every subsequence for each step $\langle k\rangle$ and the considered subsequences ends in an observation and not in an unobservable fault transition. The reading of [5] suggests that a natural perspective of this proposed paper is the Kdiagnosability.

Finally, an important difference with many papers in Petri nets [19] [7] [5] is that Section 3 exploits a series of specific count vectors developed in an approach similar to max-plus algebra where each trajectory, which is defined with respect to the time, corresponds to a unique sequence contrary to the count vector used in Section 4 where each count vector is possibly a picture of different sequences. Therefore, an estimation of subsequences is directly made.

\section{Conclusion}

In this paper, we have presented an estimation approach which generates subsequences for untimed Petri nets which can be optimal with respect to a general criterion expressing a price. The induced unobservable subnet can contain circuits and self-loops contrary to many papers in this topic.

The approach is based on two optimizations for each step, that is, the determination of a majorant of the length of the sequences (phase 1 in Section 4) and the estimation of an optimal sequence (phase 2 in Section 3). The two-phase approach needs the addition of the weak Assumption $\mathcal{A S}-5$ and we show that Assumption $\mathcal{A S}-6$ is not necessary if a time horizon can be computed. In particular, the approach is guaranteed in every situation when the induced unobservable subnet is relaxed structurally bounded which is a weak assumption introduced in this paper. We provide simple examples to illustrate the approach and show the validity of the approach.

The proposed approach avoids the classical drawback of the generation of sets which is time-costly, or even leads to a state explosion even for small Petri nets. The phase 1 where a relaxation over $\mathbb{R}$ is possible uses the standard software of linear programming as the Simplex which is efficient in practice or modern algorithms which are known to be polynomial. The phase 2 is more time costly as the resolution is over the integers but, a relaxation is also possible in the context of fault diagnosis [7]. Moreover, some general cases lead to an efficient resolution ( [13], Section IV.d in [9]) and this point will be discussed in a next paper.

As the horizon is defined by two successive occurrences of observations, a perspective is a generalization to a larger sliding horizon relevant to a subsequence of successive observations. As some firing conditions of the observations are neglected in systems (15) and (17) which are simplified forms, a second 
perspective is the generalization of these systems. Another natural perspective is to extend this technique to the schedulability analysis of P-time Petri nets and Time Stream Petri nets. Finally, the perspectives of the proposed approach are large as the technique can be applied to any problem needing the generation of sequences associated with count vectors.

\section{References}

1. A. Al-Ajeli and B. Bordbar, "Fourier-Motzkin Method for Failure Diagnosis in Petri Net Models of Discrete Event Systems," Wodes 16, Xi'an, China, May 30 - June 1, 2016.

2. F. Basile, M.P. Cabasino, and C. Seatzu, "State estimation and fault diagnosis of labeled time Petri net systems with unobservable transitions," IEEE Transactions on Automatic Control, vol. 60, no 4, pp. 997-1009, 2015.

3. F. Baccelli, G. Cohen, G.J. Olsder and J.P. Quadrat "Synchronization and Linearity An Algebra for Discrete Event Systems," Available from http://maxplus.org, New York, 1992, Wiley.

4. P. Bonhomme, "Estimation du marquage d'un réseau de Petri p-temporel sous observation partielle," Technique et Science Informatiques, vol. 35, no 4-5, p. 499-524, 2016.

5. F. Basile, P. Chiacchio and G. De Tommasi, "On K-diagnosability of Petri nets via integer linear programming," Automatica, Volume 48, Issue 9, pp. 2047-2058, 2012

6. A. Boussif, B. Liu and M. Ghazel, "Diagnosability Analysis of Intermittent Faults in Discrete Event Systems," International Journal of Control, Automation and Systems 17(X), pp. 1-14, 2019.

7. A. Chouchane, P. Declerck, A. Khedher, Atef and A. Kamoun, "Diagnostic based on estimation using linear programming for partially observable Petri nets with indistinguishable events," International Journal of Systems Science: Operations \& Logistics, pp. 1-14, Taylor \& Francis, 2018.

8. J.-C. Culioli, "Introduction à l'optimisation," Ellipses, 1994.

9. P. Declerck and P. Bonhomme, "State Estimation of Timed Labeled Petri Nets with Unobservable Transitions," IEEE Transactions on Automation Science and Engineering, Special Issue on Discrete Event Systems for Automation, Vol. 11, No. 1, ITASC9, pp. 103-110, January 2014.

10. P. Declerck and A. Guezzi, "State Estimation and Detection of Changes in Time Interval Models," Journal of Discrete Event Dynamic Systems, Vol. 24 Issue 1, pp. 53-68, March 2014

11. P. Declerck, "ARMA model and admissible trajectories in Timed Event Graphs," CESA'96, IMACS, IEEE-SMC, Lille, France, July 9-12, 1996.

12. P. Declerck, A. Chouchane and P. Bonhomme, "A strategy for Estimation in Timed Petri nets," CoDIT'17, Barcelona, Spain, April 5-7, 2017.

13. M. Dotoli, M. P. Fanti and A. M. Mangini, "Fault detection of discrete event systems by Petri nets and integer linear programming," Automatica, 45(11), pp. 2665-2672, 2009.

14. G. Jiroveanu, R. K. Boel and B. Bordbar, "On-line monitoring of large Petri net models under partially observation," Discrete Event Dynamic Systems, vol. 18, no 3, pp. 323354,2008

15. J. Komenda, S. Lahaye, J-L. Boimond and T. van den Boom, "Max-plus algebra in the history of discrete event systems," Annual Reviews in Control. 45. 10.1016/j.arcontrol.2018.04.004, 2018.

16. Lingxi Li and C. N. Hadjicostis, " Least-Cost Transition Firing Sequence Estimation in Labeled Petri Nets with Unobservable Transitions," IEEE Transactions on Automation Science and Engineering, vol. 8, no. 2, pp. 394-403, April 2011.

17. L. Libeaut and J. J. Loiseau, "Admissible initial conditions and control of timed event graphs," Proceedings of 1995 34th IEEE Conference on Decision and Control, New Orleans, LA, USA, pp. 2011-2016 vol.2, doi: 10.1109/CDC.1995.480643, 1995.

18. L. Libeaut, "Sur l'utilisation des dioides pour la commande des systèmes à événements discrets," Ph.D., Nantes, 1996. 
19. C. Mahulea, C. Seatzu, M. Cabasino and M. Silva, "Fault Diagnosis of Discrete-Event Systems Using Continuous Petri Nets," IEEE Transactions on Systems, Man, and Cybernetics, Vol.42, pp 970-984, 2012.

20. Y. Ru and C. N. Hadjicostis, "Bounds on the Number of Markings Consistent with Label Observations in Petri Nets," IEEE Transactions on Automation Science and Engineering, vol. 6, no. 2, pp. 334-344, April 2009. 